\title{
Propriedades da Madeira de Reação
}

\author{
Graziela Baptista Vidaurre ${ }^{1}$, Lucas Recla Lombardi², Leif Nutto ${ }^{3}$, \\ Frederico José Nistal França ${ }^{1}$, José Tarcísio da Silva Oliveira ${ }^{1}$, \\ Marina Donária Chaves Arantes ${ }^{1}$
}

\author{
${ }^{1}$ Departamento de Ciências Florestais e da Madeira - DCFM, Universidade Federal do Espírito Santo - UFES, \\ Jerônimo Monteiro/ES, Brasil \\ ${ }^{2}$ Empresa Klabin, Paraná, Brasil \\ ${ }^{3}$ Departamento de Engenharia e Tecnologia Florestal, Universidade Federal do Paraná - UFPR, Curitiba/PR, Brasil
}

\begin{abstract}
RESUMO
A complexidade dos processos de formação, estruturação e orientação do lenho de reação origina estudos buscando relacionar implicações tecnológicas da madeira. Em coníferas e folhosas, a madeira de reação é reflexo das diferenças químicas, físicas e anatômicas, recebendo denominações específicas. As diferentes características peculiares ao lenho de reação influenciam nas propriedades físicas, químicas, anatômicas e mecânicas, podendo limitar seu uso, com tais implicações ainda sendo ignoradas.
\end{abstract}

Palavras-chave: madeira de compressão, madeira de tração, lenho.

\section{Properties of Reaction Wood}

\begin{abstract}
The complexity of the formation, structure and orientation of reaction wood is the reason for studying the interaction between technological properties. In both softwoods and hardwoods, reaction wood reflects the chemical, physical and anatomic differences, receiving specific denominations. The specific characteristics of reaction wood influence the physical, chemical, anatomical and mechanical properties of wood and may be a limiting utilization factor - this fact is still often ignored.
\end{abstract}

Keywords: compression wood, tension wood, wood.

\section{INTRODUÇÃO}

Madeira de reação é um tecido formado tanto por efeitos genotípicos como por estímulos mecânicos; essa formação ocorre, também, pela ação da gravidade, como em situações de inclinação das árvores. Essa madeira é formada por alterações do estado fisiológico do câmbio, que provavelmente é influenciado por diferentes concentrações de substâncias de crescimento (Grosser, 1980; Sousa, 2004).
Este tipo de lenho é formado quando a árvore está sujeita a algum tipo de estresse mecânico e auxilia a planta a voltar à posição ereta. Pode ser gerado durante a vida da árvore, porém, em árvores jovens, de rápido crescimento, é mais frequente, pois estas são pequenas e mais flexíveis, podendo ser inclinadas por excesso de carga, mudanças na condição de luminosidade e ação de ventos (Mattheck \& Kübler, 1998; Oliveira et al., 2010). 
Árvores com medula excêntrica resultam de diferenças de atividade cambial, o que, consequentemente, resulta em um desenvolvimento de copa não balanceado. Quando a árvore desvia de sua posição vertical, ocorre uma reorientação gradual de suas células, a qual está associada à formação de lenho de estrutura atípica, chamado de lenho de reação (Kollman \& Côté, 1968; Dunham \& Cameron, 2000).

A madeira de reação é uma modificação da estrutura celular normal em plantas lenhosas, que pode variar de uma condição complexa a uma mudança pouco percebida nas características das células. A natureza e a aparência da madeira de compressão são similares em todas as espécies de coníferas. Por outro lado, a madeira de tração é menos uniforme em aparência e varia principalmente em sua natureza, entre espécies e gêneros, sendo que poucos gêneros de folhosas, como Drimys, Buxus e Cotinus, produzem lenho de reação semelhante, em localização e aparência, às madeiras de compressão (Panshin \& De Zeeuw, 1980).

A presença de tecidos de reação, por menor que seja sua quantidade, causa mudanças significativas nas propriedades da madeira e, consequentemente, na qualidade desta, o que de fato fundamenta a classificação da madeira de reação como um defeito. Esse fato é comprovado pela aplicação de normas para classificar madeira para produtos sólidos na Europa, América do Norte, Ásia e na Oceania, tanto a sua forma roliça como a serrada. A tentativa de eliminar toras ou madeira serrada com madeira de reação demonstra que os problemas causados pela mesma são bem conhecidos pela indústria. A eliminação de toras com madeira de reação embasa-se na excentricidade da medula, tanto para folhosas quanto em coníferas, mas não é infalível. Para madeira serrada, existem normas somente para coníferas, que possuem uma coloração mais escura.

As tensões de crescimento podem estar ou não relacionadas com lenho de reação. As tensões de crescimento ocorrem em árvores tanto em coníferas como em folhosas, sendo que as folhosas desenvolvem níveis superiores de tensões de crescimento.

Latorraca \& Albuquerque (2000) afirmaram que espécies de folhosas, como o eucalipto, são mais propensas a desenvolver altos níveis de tensões de crescimento, por causa da presença de lenho juvenil, que possui massa específica mais baixa, menores diâmetros de fuste e maior proporção de lenho de reação.

Em árvores do gênero Eucalyptus, um indicativo da presença de lenho de tração é a elevada retratibilidade e maior densidade básica quando comparada a madeira normal (Monteiro et al., 2010).

Tendo em vista a importância da qualidade da madeira para seu uso racional e sustentável, este trabalho trata de uma revisão bibliográfica acerca dos efeitos do lenho de reação nas propriedades tecnológicas da madeira.

\section{LENHO DE TRAÇÃO}

O lenho de reação das folhosas, chamado de lenho de tração, caracteriza-se por alto conteúdo de celulose e baixo conteúdo de lignina, quando comparado com o lenho normal. As fibras do lenho de tração possuem uma espessa camada gelatinosa na parte interna à parede celular secundária (Bailléres et al., 1997).

Em espécies tropicais há evidências de que a madeira de tração possa se formar em árvores não inclinadas, como um meio da copa se movimentar no espaço para obter luz suficiente em florestas com espaçamento mais denso (Timell, 1986; Warenjö, 2003)

Entretanto, a localização da madeira de tração nem sempre ocorre na região de crescimento acelerado, podendo ocorrer no lado suprimido, como em galhos de madeira de Sassafras albidum e em tronco de madeira de Tilia americana. Em muitas espécies, a madeira de tração pode ser formada com pouca evidência de excentricidade no tronco maduro, portanto a irregularidade da seção transversal não é uma indicação constante da presença desse tipo de madeira de reação. Esse fato é particularmente evidente em alguns gêneros, como Paulowniae Catalpa, e em raízes que evidenciam a madeira de tração sem nenhuma orientação evidente em relação ao eixo gravitacional (Panshin \& De Zeeuw, 1980).

Macroscopicamente, a madeira de tração é mais clara e brilhante, porém tal característica só é reconhecível em madeira recém-cortada; em madeira seca é detectada por meio de microscopia. A 
mais típica delas é o aparecimento de uma superfície lanosa em madeiras serradas verdes (Badia et al., 2005; Grosser, 1980). O aspecto áspero de tábuas serradas de madeiras de folhosas recém-derrubadas está relacionado com a presença do lenho de tração, o que oferece dificuldades durante o desdobro e a secagem, e nos estágios posteriores de acabamento - por exemplo, no aplainamento. Tarmian et al. (2009) ressaltaram que, na secagem de madeira de reação, defeitos como rachaduras e empenamentos são comuns.

$\mathrm{Na}$ madeira de Ficus sp., por exemplo, as extremidades das fibras presentes em lenhos de tração não proporcionam cortes com superfície de brilho prateado, que contrasta com o tecido circundante. Outros tipos de madeiras fornecem indicação de zonas da madeira de tração na seção transversal polida, em função da cor mais escura do que a madeira normal dessas regiões (Kollman \& Côté, 1968; Panshin \& De Zeeuw, 1980).

\subsection{Características microscópicas}

As principais diferenças anatômicas associadas ao lenho de tração estão relacionadas às fibras. A espessura da parece celular é maior no lenho de tração; contudo Souza (2004) ressaltou que a largura das fibras não é um indicador de presença de lenho de reação em folhosas, pois não ocorreu diferença significativa nesse parâmetro em comparação com a madeira normal para a madeira de E. grandis com 4 anos e 11 meses de idade.

$\mathrm{O}$ autor ressaltou ainda que para o comprimento das fibras de tração foi significativamente maior que a madeira normal. Ferreira (2007) estudando clones de Eucalyptus spp com 2 anos de idade observou que não houve diferença significativa para o comprimento de fibras de lenho de tração e lenho normal.

Heinrich \& Gärtnert (2008) submeteram árvores de Alnusglutinosa e Fagussylvatica a diferentes inclinações de tronco e copa e analisaram as propriedades anatômicas da madeira de tração. Observaram que o aumento do ângulo de inclinação do tronco implicou no aumento da tensão, gerando uma redução da área dos lúmens dos vasos. Além disso, os autores mencionaram que a utilização de técnicas anatômicas, associadas com a dendrogeomorfologia e o estudo de anéis de crescimento, pode suplementar informações sobre o tipo e a intensidade de fatores geradores de lenho de tração durante o crescimento das árvores. Os vasos em zonas definidas de madeira de tração são de menor diâmetro e menos numerosos, quando comparados aos de madeira normal (Morey, 1980; Jin \& Kwon, 2009).

Anatomicamente, as fibras da madeira de tração típicas diferem das fibras da madeira normal no conteúdo de uma camada na parede celular, referida como camada G, que consiste principalmente de celulose. Essa camada, compacta e contínua, localiza-se no lado interno da célula voltada para o lúmen, podendo ser depositada de diferentes maneiras (Kollman \& Coté, 1968; Tissoumis, 1978; Peña \& Peris, 1996). No passado acreditava-se que a camada gelatinosa era deslignificada, mas o uso de novas técnicas químicas comprovaram a presença de quantidades de pectina, hemiceluloses e lignina na camada $G$ de Liquidambar styraciflua e Celtis occidentalis (Bowling \& Vaughn, 2008).

Clair et al. (2010) sugeriram que a parte interna da camada S2 da parede celular contribui significativamente para a geração de tensão em madeira de tração de híbrido de Populus deltoides x Populus trichocarpa, sendo que a tensão de crescimento ocorre com ou sem a presença camada G.

Todas as angiospermas aparentemente são capazes de produzir madeira com elevadas quantidades de madeira de tração (Clair et al., 2006). Em algumas espécies, a diferença na estrutura da fibra é evidente, com a presença da camada $G$ na madeira de tensão. Em outros casos, a diferença entre a madeira normal e a com elevada tensão não é tão evidente à partir de observações em anatomia clássica.

Em alguns gêneros, como Fraxinus, Lagunaria e Tilia, as fibras gelatinosas são raras ou nunca formadas (Timell, 1969). O uso de luz polarizada, raios $\mathrm{X}$ e microscopia eletrônica tem evidenciado que as microfibrilas de celulose da camada gelatinosa são altamente cristalinas e axialmente orientadas, paralelamente à fibra (Mia, 1968 apud Jin e Kwon, 2009). 
Clair et al. (2006), estudando 21 espécies de angiospermas tropicais por meio de técnicas anatômicas, observaram que em 14 espécies a camada $\mathrm{G}$ foi ausente, ressaltaram que é fundamental a realização de testes mais específicos para a detecção da camada $G$ e afirmaram que a ausência dessa camada não indica a ausência de tecido de reação.

Segundo Panshin \& De Zeeuw (1980) e Jin \& Kwon (2009), a organização da parede celular pode variar, sendo que a camada gelatinosa pode estar presente em fibras de madeira de tração, em adição às três camadas da parede celular secundária lignificada normalmente. Em outros casos, a camada $G$ pode substituir a camada $\mathrm{S}_{3}$ em fibras de madeira de tração, com as regiões $S_{1}$ e $S_{2}$ da parede secundária normal permanecendo inalteradas; por fim, as camadas de parede podem ser reduzidas a uma espessa camada gelatinosa e à zona que corresponde à camada $\mathrm{S}_{1}$ de fibras normais.

Por muito tempo, se acreditou que a camada $G$ era composta apenas por celulose cristalina; contudo, Bowling \& Vaughn (2008) ressaltam que, utilizandose técnicas imunocitoquímicas, determinou-se a presença de proteínas arabinogalactanas - AGPs e ramnogalactouranos I - RG I na madeira de tração de Liquidambar styraciflua e Celtis occidentalis, obtidas de florestas experimentais no estado do Mississipi, Estados Unidos. Os autores ainda afirmaram que a presença de ramnogalactouranos I pode estar associada com a tensão criada na camada G, uma vez que esse componente é predominante na composição do açucararabinose, o qual é altamente hidrofílico.

\subsection{Composição química}

Timell (1969) mencionou que a camada gelatinosa consiste somente de celulose, porque, com essa composição, tenta-se estabilizar o baixo conteúdo de lignina da madeira de tração, que é causado por uma redução no teor de lignina de cada fibra ou é o resultado de uma introdução extra de celulose.

Em madeiras de tração, há uma lignificação variável das fibras. A camada $G$ é levemente lignificada, possuindo um alto conteúdo de celulose, baixo conteúdo de lignina, maior quantidade de galactanas e menor quantidade de xilanas do que o normal; no entanto, há uma variação desses constituintes entre as espécies (Sousa, 2004).

$\mathrm{O}$ amido e os açúcares armazenados no tecido da madeira de tração estão presentes em quantidades menores do que no lado oposto da mesma seção transversal, na área de crescimento suprimido. Tal comportamento poderia afetar a extensão de ataques biológicos por organismos que dependem dessas sustâncias para seu desenvolvimento ou sua sobrevivência (Panshin \& De Zeeuw, 1980).

A distribuição de lignina dentro da parede celular de fibras de madeiras de tração foi estudada por meio de corantes indicadores de microscopia de contraste de fase. Usando-se essas técnicas, pode-se concluir que a lignina está ausente na camada gelatinosa e que pode ser menos presente ou ausente em uma ou mais das outras camadas da parede secundária. Essa deficiência geral de lignina nas paredes secundárias de fibras é a característica principal do desenvolvimento de madeira de tração em folhosas (Panshin \& De Zeeuw, 1980; Latorraca \& Albuquerque, 2000).

Bleile (2006) pôde demonstrar a dominância de celulose na madeira de reação de Fagus silvatica por meio da aplicação de ácido clorídrico $(\mathrm{HCl})$, que reage somente com a lignina. Enquanto a madeira normal evidenciou uma coloração vermelha, a madeira de reação possui cor esbranquiçado, indicando uma aglomeração de células com alto conteúdo de celulose, o que podia ser comprovado por meio de uma análise microscópica da madeira dessa zona. $\mathrm{O}$ mesmo teste não funcionou para a madeira de Notogfagus betuloides (Manso, 2003), pois a análise microscópica demonstrou que a espécie analisada também forma células com uma camada $G$ de alto teor de celulose, mas que estas aparecem de forma misturada com madeira normal dentro da zona de madeira de reação, que reage com o ácido clorídrico.

Em estudos com quatro espécies de folhosas Acacia salicina, Albizzia lebbeck, Tamarix aphylla e Pithecellobium dulce -, todas com 12 anos de idade, Abdel-Aal et al. (2011) comprovaram que a ocorrência do lenho de tração influencia na composição química da madeira. Foram encontradas maiores quantidades de celulose e menores valores de hemicelulose, lignina, extrativos e cinzas para 
madeiras de tração em comparação a madeiras normais (Tabela 1 ).

Bailléres et al. (1997) observaram que no lenho de tração das madeiras do gênero Buxus, o teor de lignina compõe-se levemente enriquecido em unidades de guaiacil, comparada com a lignina da madeira normal. Contudo, o enriquecimento em lignina é mais pronunciado no caso de madeira de compressão em gimnospermas.

Timel (1969) analisou três espécies de eucalipto, destacando-se o teor de lignina, uma vez que este foi, em média, 8,75\% inferior no lenho de tração, quando comparado ao lenho de madeira normal (Tabela 2).

Aguayo et al. (2010), estudando Eucalyptus globulus de 8 anos, oriundo da região de Bio Bio, Chile, encontraram redução significativa de $4 \%$ no conteúdo de lignina da madeira de tração, comparado ao da madeira normal. A quantidade de lignina do tipo siringil da região tracionada foi
$15 \%$ maior que a encontrada no lenho oposto, não havendo diferenças significativas para as quantidades de lignina guaiacil.

Wang et al. (2010) encontraram valores maiores para tensão de crescimento no lado superior de galhos de Viburnum odoratissimum. O lenho do lado superior dos galhos apresentou menor rigidez e maior viscosidade devido a variações dos teores de siringil e guaiacil na lignina do lenho, aumentando a elasticidade.

Quanto aos extrativos, Sousa (2004) obteve conteúdos da ordem de 1,69\% para madeiras normais e de $1,22 \%$ para madeiras de tração em E. grandis, plantado no Estado de Minas Gerais, indicando que esse parâmetro químico também sofre influencia da madeira de tração.

Testes de polpação kraft demonstraram que rendimentos de polpa química são maiores quando provenientes de madeira de tração do que de

Tabela 1. Composição química da madeira de tração e normal de folhosas (\%).

Table 1. Chemical composition of reaction and normal wood of hardwoods (\%).

\begin{tabular}{|c|c|c|c|c|}
\hline Espécies & Celulose & Hemicelulose & Lignina & Cinzas \\
\hline \multicolumn{5}{|c|}{ Acacia salicina } \\
\hline $\mathrm{MT}^{*}$ & 48,35 & 21,39 & 30,25 & 1,07 \\
\hline $\mathrm{MN}^{*}$ & 46,82 & 22,72 & 31,00 & 1,09 \\
\hline \multicolumn{5}{|c|}{ Albizzia lebbeck } \\
\hline MT & 47,17 & 23,72 & 29,41 & 0,93 \\
\hline $\mathrm{MN}$ & 41,43 & 26,02 & 32,54 & 1,30 \\
\hline \multicolumn{5}{|c|}{ Tamarix aphylla } \\
\hline MT & 44,15 & 22,82 & 33,20 & 3,95 \\
\hline $\mathrm{MN}$ & 42,45 & 23,09 & 34,02 & 5,96 \\
\hline \multicolumn{5}{|c|}{ Pithecellobium dulce } \\
\hline MT & 47,22 & 22,68 & 29,79 & 1,31 \\
\hline $\mathrm{MN}$ & 44,71 & 24,52 & 31,02 & 1,52 \\
\hline
\end{tabular}

MT = madeira de tração; $\mathrm{MN}$ = madeira normal. Fonte: Adaptado de Abdel-Aal et al. (2011).

Tabela 2. Composição química da madeira normal e da madeira de tração de eucalipto.

Table 2. Chemical composition in normal wood and tension wood of Eucalytus.

\begin{tabular}{|c|c|c|c|c|c|c|c|}
\hline \multirow{2}{*}{ Espécies } & \multicolumn{2}{|c|}{ Lignina (\%) } & \multicolumn{2}{|c|}{ Celulose (\%) } & \multicolumn{2}{|c|}{ Pentosanas (\%) } & \multirow{2}{*}{ Autor } \\
\hline & Normal & Tração & Normal & Tração & Normal & Tração & \\
\hline E. camaldulensis & 22,7 & 15,2 & & & & & \multirow{4}{*}{ Timell (1969) } \\
\hline E. goniocalix & 23,2 & 13,8 & 42,9 & 62,1 & 19,3 & 11,0 & \\
\hline E. regnans & 22,2 & 16,0 & & & & & \\
\hline E. regnans & 25,1 & 13,2 & & & & & \\
\hline E. grandis & 29,72 & 23,9 & & & & & Sousa (2004) \\
\hline E. globulus & 26,9 & 21,4 & 44,9 & 45,1 & 23,0 & 24,1 & Aguaio et al. (2010) \\
\hline
\end{tabular}


madeira normal e que a madeira de tração pode ser mecanicamente desfibrada com mais facilidade do que a madeira normal (Sousa, 2004). Contudo, o papel produzido com fibras de madeira de tração tem uma resistência menor que o papel de fibras de madeira normal. A madeira de tração é adequada para polpas solúveis, uma vez que o rendimento é alto e a resistência da fibra original não é fator limitante nesses tipos de polpas (Panshin e De Zeeuw, 1980). Sousa (2004) também observou maior rendimento (9\%) na polpação química da madeira de Eucalyptus grandis, comparando à madeira normal, além de menor consumo de reagentes na polpação.

\subsection{Propriedades físicas e mecânicas}

O lenho de tração de Eucalyptus globulus com 11 anos de idade apresentou baixa correlação com a densidade básica da madeira; contudo, amostras com elevadas quantidades de lenho de tração tenderam a apresentar maiores densidades básicas e, assim, aumentar a incidência de contrações na madeira serrada (Washusen et al., 2001).

Washusen (2000) ressaltou que o pequeno acréscimo na densidade básica causado pela presença do lenho de tração pode interferir na classificação de toras, uma vez que em usos estruturais é comum a classificação embasada na densidade, visto a correlação entre densidade básica e resistência mecânica.

Sousa (2004) relatou em seu estudo que, para a madeira de tração em Eucalyptus grandis, não ocorreu diferença significativa da densidade básica no sentido medula-casca; contudo, analisando apenas discos da região superior do tronco, o autor encontrou diferenças de 0,017 g.cm-3, sendo tais valores significativos para a região estudada. Pôdese inferir, dessa forma, que a presença do lenho de tração é mais significativa na parte superior da árvore, fato comprovado pela análise de cortes histológicos.

Ao estudar a madeira de eucalipto de quatro diferentes espécies - E. pilularis, E. maculata, E. urophylla e E. camaldulensis - com 32 anos de idade, cultivadas em região com declividade, Monteiro et al. (2010) relataram que contrações tangenciais e volumétricas no lenho de reação e no lenho normal não diferiram estatisticamente. Souza (2004) encontrou diferença significativa para valores de contração volumétrica para madeira de Eucalyptus grandis.

$\mathrm{Na}$ Tabela 3 pode-se visualizar algumas propriedades mecânicas da madeira de tração. A compressão paralela à grã, a compressão perpendicular à grã, o módulo de ruptura em flexão estática, o cisalhamento longitudinal e o módulo de elasticidade em flexão apresentaram menores valores médios do que madeira de tração, em todos os níveis de umidade, em relação à madeira normal. Quanto à tração paralela à grã, as evidências indicam uma menor resistência da madeira de tração em relação à madeira normal na condição verde, porque, nessa condição de umidade, a camada gelatinosa presente nessas madeiras não está bem ligada ao resto da parede secundária, influenciando assim a resistência. Por outro lado, para uma condição de $15 \%$ de umidade, observa-se aumento de resistência para madeira de tração de Populus regenerata (Tabela 3).

Tabela 3. Propriedades físicas de madeira de tração e madeira normal.

Table 3. Physical and mechanical properties oftension wood and normal wood.

\begin{tabular}{|c|c|c|c|c|c|c|c|c|}
\hline \multirow{2}{*}{$\begin{array}{l}\text { Madeira de } \\
\text { tração }\end{array}$} & \multicolumn{2}{|c|}{ Acer saccharum } & \multicolumn{2}{|c|}{ Betula pubescens } & \multicolumn{2}{|c|}{ Populus deltoides } & \multicolumn{2}{|c|}{ Populus regenerata } \\
\hline & MT & MN & MT & $\mathbf{M N}$ & MT & $\mathbf{M N}$ & MT & MN \\
\hline Teor de umidade (\%) & 10 & 10 & 36 & 36 & 12 & 12 & 15 & 15 \\
\hline Densidade aparente $\left(\right.$ g. $\left.\mathrm{cm}^{-3}\right)$ & - & - & 0,69 & 0,64 & 0,44 & 0,43 & 0,44 & 0,39 \\
\hline Fator Anisotrópico & 1,12 & 1,72 & 1,53 & & - & - & - & - \\
\hline Módulo de ruptura (MPa) & 102,94 & 122,41 & - & - & - & - & - & - \\
\hline Módulo de Elasticidade (MPa) & 10.866 & 12.838 & - & - & - & - & - & - \\
\hline Compressão paralela (MPa) & 48,81 & 52,74 & 19,80 & 22,65 & 32,43 & 41,80 & - & - \\
\hline Tração paralela (MPa) & - & - & - & - & - & - & 103,42 & 65,5 \\
\hline
\end{tabular}

Fonte: Adaptado de Panshin \& De Zeeuw (1980). MT = madeira de tração; MN = madeira normal. 
As evidências contraditórias na resistência à tração baixa de madeira de tração são devidas ao fato de que essas madeiras possuem um defeito chamado de cerne quebradiço.

As resistência das madeiras de tração, em comparação à madeira normal, podem ser explicadas qualitativamente com base na mudança das taxas de celulose-lignina. Uma diminuição no teor de lignina na madeira de tração indica que há um decréscimo no apoio lateral entre as microfibrilas de celulose na parede celular. Como resultado, as microfibrilas e as lamelas na madeira de tração agem como longas colunas sem apoio sob compressão paralela à grã e empenam sob cargas menores do que ocorre com madeira normal, que contém mais lignina. $\mathrm{O}$ incremento na resistência à tração na madeira de tração seca também está relacionado ao aumento do teor de celulose em relação à madeira normal (Kollman \& Coté, 1968; Panshin \& De Zeeuw, 1980).

\section{LENHO DE COMPRESSÃO}

A madeira de compressão em toras, geralmente, é indicada pela formação de anéis de crescimento excêntricos, que parecem conter uma proporção maior de lenho tardio na região de crescimento rápido. Como regra geral, o brilho da madeira de compressão em superfícies longitudinais é menos evidente do que aquele exibido pela madeira normal. Como regra geral, o brilho da madeira de compressão em superfícies longitudinais é menos evidente do que aquele exibido pela madeira normal (Panshin \& De Zeeuw, 1980).

Contudo, a presença do lenho de compressão, mesmo em condições extremas, pode não ser acompanhada de excentricidade evidente na seção transversal (Tarmian et al., 2009). Em estudo com Piceaabies, Warensjö (2003) observou a presença de lenho de compressão entre estreitos anéis de crescimento, não comprometendo a excentricidade da medula.

A densidade é quase que invariavelmente alta em madeira de compressão e está associada com propriedades mais baixas de resistência do que aquela que seria prevista com base em seu peso. A madeira de compressão bem desenvolvida geralmente é mais densa do que o esperado para uma dada espécie e é mais difícil de ser trabalhada com ferramentas do que a madeira normal (Zobel \& Van Buijtenen, 1989; Peña \& Peris, 1996).

\subsection{Características microscópicas}

Anatomicamente, o lenho de compressão caracteriza-se por anéis de crescimento constituídos de traqueídeos arredondados em seção transversal, em contraste com o desenho angular dos traqueídeos normais, sendo essa característica mais evidente no lenho tardio.

A espessura da parede dos traqueídeos de madeira de compressão no lenho tardio é, aproximadamente, duas vezes maior que em traqueídeos normais. Os traqueídeos de lenho inicial têm aproximadamente as mesmas espessuras da parede das células normais. Os diâmetros das células radiais apresentam pouca diferença, exceto nos limites dos anéis de crescimento. Tanto o diâmetro médio radial quanto o tangencial são menores do que aqueles que seriam evidentes em uma madeira normal (Panshin \& De Zeeuw, 1980).

Pelz (2002) encontrou microfissuras nas paredes celulares da madeira de compressão de Larix decidua (Mill.). Em razão do alto teor de lignina das paredes, as mesmas demonstraram uma dureza elevada, mas uma elasticidade baixa.

A camada $\mathrm{S}_{2}$ modificada em madeira de compressão possui ângulo microfibrilar de cerca de $45^{\circ}$ em relação ao eixo da célula. Esse ângulo é maior que o encontrado na camada $S_{2}$ da madeira normal. O ângulo espiral é evidente nas paredes celulares de muitas espécies de coníferas, visto a presença de padrões helicoidais que acompanham o ângulo microfibrilar. Esses padrões são, na realidade, um sistema de cavidades radiais que se originam na camada $S_{2}$, durante a formação dessa camada da parede, e não são causadas pela secagem da parede celular (Bamber, 2001; Klock et al., 2005).

TomazeloFilho(1987), estudandoas propriedades anatômicas da madeira de Pinus oocarpa com 11 anos de idade, observou que, os traqueídeos do lenho de compressão foram $15 \%$ menores que o lenho normal, a largura do traqueídeo foi $8 \%$ menor, o diâmetro do lúmen foi $15 \%$ menor e a espessura da parede celular foi $14 \%$ maior. 
Tarmian et al. (2009), analisando a secagem da madeira de compressão de Picea abies em estufa, observaram que a elevada espessura da parede celular dos traqueídeos do lenho de compressão comprometeu as taxas de secagem, sendo necessárias taxas de secagem menores. Além disso, a presença de pontoações maiores e mais numerosas na madeira oposta ao lenho de compressão proporcionou a secagem mais rápida da madeira.

\subsection{Composição química}

De modo geral, o teor médio de lignina, com base no peso seco, cresce cerca de $9 \%$, o teor de celulose em madeira de compressão é cerca de 10\% menor, em relação aos níveis normais, e o teor de galactose é $7,8 \%$ maior que o da madeira normal.

Estudos das estruturas dos traqueídeos de madeira de compressão evidenciaram que a lamela média composta é similar à da madeira normal; a camada $S_{1}$ é mais espessa e tem a mesma organização microfibrilar, mas seu teor de lignina é menor. A distribuição de lignina está concentrada na camada $\mathrm{S}_{2}$ (Kollman \& Coté, 1968; Panshin \& De Zeeuw, 1980).

A análise da celulose em madeira de compressão identificou que esta tem grau mais baixo de cristalinidade e que alguns componentes das hemiceluloses são retidos na celulose purificada.
Timell (1986) relatou que cerca de metade da galactose é retida na celulose alfa-purificada.

O mesmo autor relatou que a madeira de compressão de quatro espécies de gimnospermas contém consideravelmente mais lignina do que a madeira normal correspondente (Tabela 4). Além disso, observou-se significativa diferença para os teores de celulose e extrativos, de modo que, para a celulose, a diferença máxima observada foi de $18,40 \%$ na madeira de Picea abies.

Morohoshi \& Sakakibara (1971) e Yasuda \& Sakakibara (1975) encontraram, para madeira de compressão de Abies sachalinensis e Larix leptolepis, 35,3 e 39,3\% de teores de lignina, respectivamente, enquanto em madeira normal esses valores são 28,3 e $28,1 \%$.

Comparando-se os constituintes químicos na madeira de compressão de Pinus radiata, plantados no Chile, com a madeira normal, Diaz-vaz et al. (2009), encontraram teor de lignina no lenho de compressão $1,94 \%$ maior que no lenho oposto e o conteúdo de celulose no lenho de compressão foi $22 \%$ menor, seguindo o encontrado por Timell (1986) e as mudanças na composição química da madeira de compressão podem interferir nos usos para produção de painéis, celulose, papel, secagem e produtos sólidos, pois tal fato pode reduzir a sua adequabilidade a esses fins.

Tabela 4. Conteúdo de celulose, lignina (Klason), pentosanas e extrativos (álcool-benzeno) da madeira normal e de compressão das seções transversais de quatro espécies de coníferas.

Table 4. Cellulose content, lignin (Klason), pentosans and extractives (ethanol-benzene) of normal and compression wood of the cross sections of four softwoods species.

\begin{tabular}{|c|c|c|c|c|}
\hline Espécies & Celulose & Lignina & Pentosana & Extrativos \\
\hline Abies alba & \multicolumn{4}{|c|}{$\%$} \\
\hline $\mathrm{MN}$ & 56,4 & 28,6 & 12,6 & 2,25 \\
\hline MC & 47,8 & 34,4 & 13,6 & 2,43 \\
\hline \multicolumn{5}{|l|}{ Larix decidua } \\
\hline $\mathrm{MN}$ & 56,4 & 27,3 & 11,6 & 1,00 \\
\hline MC & 49,7 & 35,2 & 11,1 & 3,59 \\
\hline \multicolumn{5}{|l|}{ Picea abies } \\
\hline $\mathrm{MN}$ & 62,2 & 27,9 & 10,6 & 5,63 \\
\hline MC & 43,8 & 34,9 & 11,7 & 6,66 \\
\hline \multicolumn{5}{|l|}{ Pinus nigra } \\
\hline $\mathrm{MN}$ & 60,8 & 26,7 & 11,0 & 2,45 \\
\hline $\mathrm{MC}$ & 48,2 & 36,9 & 11,8 & 2,75 \\
\hline
\end{tabular}

Fonte: Adaptado de Timell (1986). MN = madeira normal; $\mathrm{MC}=$ madeira de compressão. 
Tabela 5. Propriedades físicas e mecânicas de madeira de compressão e de madeira normal.

Table 5. Physical and mechanical properties ofcompression wood and normal wood.

\begin{tabular}{|c|c|c|c|c|c|c|}
\hline \multirow{2}{*}{$\begin{array}{l}\text { Madeira de } \\
\text { compressão }\end{array}$} & \multicolumn{2}{|c|}{ Pinus ponderosa } & \multicolumn{2}{|c|}{ Pseudotsuga menziessii } & \multicolumn{2}{|c|}{ Sequoia sempervirens } \\
\hline & MC & MN & MC & MN & MC & $\mathbf{M N}$ \\
\hline Teor de umidade (\%) & 12,6 & 12 & 12,1 & 11,5 & 10,5 & 9,9 \\
\hline Densidade aparente $\left(\mathrm{g} \cdot \mathrm{cm}^{-3}\right)$ & 0,499 & 0,372 & 0,527 & 0,459 & 0,51 & 0,38 \\
\hline Fator Anisotrópico & 2,31 & 1,64 & 1,68 & 1,73 & 1,71 & 2,33 \\
\hline Módulo de ruptura (MPa) & 80,73 & 67,84 & 86,18 & 89,28 & 61,29 & 70,40 \\
\hline Módulo de Elasticidade (MPa) & $7.025,75$ & $9.273,45$ & $8.190,97$ & $11.486,66$ & $5.433,06$ & $8.639,13$ \\
\hline Compressão paralela (MPa) & 41,16 & 35,92 & 49,22 & 49,85 & 49,98 & 49,36 \\
\hline Tração paralela (MPa) & - & - & 88,25 & 91,01 & 52,12 & 61,01 \\
\hline
\end{tabular}

Fonte: Adaptado de Panshin \& De Zeeuw (1980). MC = madeira de compressão; $\mathrm{MN}=$ madeira normal.

\subsection{Propriedades físicas e mecânicas}

A característica física mais evidente da madeira de compressão é o aumento da massa específica acima daquela da madeira normal equivalente (Tomazello Filho \& Silva, 1987). Diaz-vaz et al. (2009) observaram valores de densidade superiores em $8 \%$ na madeira de compressão em relação à madeira normal para a madeira de Pinus radiata.

$\mathrm{O}$ aumento na densidade deve-se às paredes geralmente mais espessas na madeira de compressão, o que resulta em cerca de um terço a mais de material de parede celular do que na madeira normal (Panshin \& De Zeeuw, 1980).

A contração e o inchamento da madeira de compressão são maiores do que os da madeira normal, como pode ser visualizado na Tabela 5. O aumento de contração ao longo da grã é elevado, sendo relatado por Panshin \& De Zeeuw (1980) como $8,6 \%$. Esse aumento é acompanhado pela redução da contração lateral, com mudanças radiais e tangenciais na madeira de compressão de cerca da metade daquelas da madeira normal (Tabela 5). A contração excessivamente grande ao longo da grã faz com que tábuas que contenham madeira de compressão empenem e se torçam fortemente, o que causa rachaduras frequentes nas junções entre a madeira de compressão e a normal. Essas características alteradas podem ser explicadas com base no aumento no ângulo microfibrilar na camada $\mathrm{S}_{2}$ da parede celular da madeira de compressão, o que promove um maior componente de mudança na dimensão (Albuquerque \& Latorraca, 2000).
As propriedades de tração e o módulo de elasticidade da madeira de compressão são menores do que o esperado, com base em sua massa específica. A baixa resistência à tração ao longo do eixo da célula pode ser explicada pela presença de cavidades espiraladas na parede celular dos traqueídeos do lenho de compressão (Timell, 1986).

\section{CONSIDERAÇÕES FINAIS}

O lenho de reação das folhosas é caracterizado por possuir alto conteúdo de celulose e baixo de lignina, quando comparado com o da madeira normal equivalente. Anatomicamente, as fibras de madeira de tração diferem das fibras da madeira normal no conteúdo da parede celular referida com camada gelatinosa.

Anatomicamente, as fibras de madeira de tração diferem das fibras da madeira normal no conteúdo de uma camada na parede celular referida como camada gelatinosa, que consiste basicamente de celulose.

Quanto ao processamento mecânico, a madeira de tração confere características que dificultam o desdobro primário e os estágios posteriores de acabamento, por liberar tensões internas nas peças que podem causar empenamentos e rachaduras. Além disso, podem propiciar defeitos de secagem nas peças por processos de contração heterogênea. Assim, o grau de aproveitamento vai reduzido e a madeira geralmente é classificada como de valor mais baixo. Em muitos casos, o lenho de reação causa defeitos secundários no material processado. 
A presença da camada $G$ proporciona alterações significativas na composição química da madeira, bem como em outras propriedades relacionadas. Enquanto a camada G é encontrada na maioria das espécies de folhosas, a composição das células do lenho de tração ocorre de forma aglomerada, formando zonas de células puras dentro do lenho de tração (Fagus sp.); em outras, somente dentro de um anel de crescimento ou misturadas com células de lenho normal (Nothofagus, Eucalyptus). No último caso, os efeitos negativos de uma contração heterogênea durante a secagem são reduzidos, mas também não é possível detectar zonas de madeira de tração em um corte transversal (disco) com o uso de reagentes químicos. Dessa forma, altos teores de celulose e uma redução da lignina, além de hemiceluloses, são índices predominantes na análise química de madeira de tração. O teor de extrativos é variável, não sendo observada uniformidade entre espécies.

A densidade básica não é um bom parâmetro para distinção de lenho de tração e lenho normal, pois a densidade está diretamente ligada à espessura de parede celular e o lenho de tração não tem influência significativamente na parede celular. Quimicamente a camada $G$ proporciona aumento do teor de celulose e redução do teor de lignina, e o peso molecular da lignina é superior ao da celulose, balanceando a relação massa lignina $\mathrm{x}$ massa de celulose.

O uso de madeira de tração na polpação química interfere em: consumo de químicos do cozimento; rendimento do processo; qualidade final da polpa, e consequentemente do papel. São observados resultados positivos para rendimentos de polpa química de eucalipto com madeira de tração, mas resultados negativos predominam nas análises de propriedades do papel, bem como no aumento do consumo de álcali.

O conteúdo de lignina do lenho de compressão é maior e o de celulose é menor quando comparado com o lenho de madeira normal equivalente.

Os anéis de crescimento do lenho de compressão consistem de traqueídeos arredondados e, consequentemente, possuem espaços intercelulares maiores, sendo as camadas da parede secundária reduzidas às camadas $S_{1}$ e $S_{2}$ modificadas.
O beneficiamento da madeira de compressão para produtos sólidos geram defeitos no desdobro e secagem, visto a maior contração e inchamento do lenho. O lenho de compressão é visível, tanto no corte transversal (testa de troncos) como na madeira serrada (zonas de coloração escura intensa), facilitando a classificação visual da madeira de reação nas coníferas. Tal indicativo foi incluído na maioria das normas de classificação de madeira roliça e madeira serrada.

A identificação e a caracterização macroscópica de lenho de tração não são tão explícitas como em lenho de compressão. É necessário o uso de técnicas como: microscopia, difração de raios $\mathrm{X}$ e espectroscopia de infravermelho. Ressaltase a dificuldade na precisão da determinação e quantificação deste tecido.

Apesar de o lenho de compressão possuir maior massa específica, as propriedades mecânicas possuem menor resistência. Essa característica causa problemas na classificação de madeira serrada com equipamentos usados na classificação mecanizada ou semimecanizada, que estimam a classe de resistência para fins de construção por meio da correlação entre densidade e módulo de ruptura. Tal fato está relacionado às propriedades químicas, uma vez que o aumento do percentual de lignina contribui significativamente para a perda de resistência da parede celular.

\section{STATUS DA SUBMISSÃO}

Recebido: 24/01/2012

Aceito: 25/05/2012

Publicado: 28/02/2013

\section{AUTOR(ES) PARA CORRESPONDÊNCIA}

\section{Graziela Baptista Vidaurre}

Departamento de Ciências Florestais e da Madeira - DCFM, Universidade Federal do Espírito Santo - UFES,

Av. Governador Lindenberg, 316, CEP 29550-000, Jerônimo Monteiro, ES, Brasil e-mail: graziela@cca.ufes.br 


\section{REFERENNCIAS}

Abdel-AalMA,NasserRA,Al-MefarrejHA.Comparative Study on Tension and Opposite woods of some species grown under Saudia Arabia condition. Middle-East Journal of Scientific research 2011; 7(4): 490-496.

Aguayo MG, Quintupill L, Castillo R, Baeza J, Freer J, Mendonça RT. Determination of differences in anatomical and chemical characteristics of tension and opposite wood of 8-year old Eucalyptus globulus. Maderas, Ciencia y Tecnología 2010; 12(3): 241-252.

Albuquerque CEC, Latorraca JVF. Influência das características anatômicas da madeira na penetração e adesão de adesivos. Floresta $e$ Ambiente 2000; 7(1): 158-166.

Badia MA, Mothe F, Constant T, Nepveu G. Assessment of tension wood detection based on shiny appearance for three poplar cultivars. Forest Science 2005; (62): 43-49.

Bailléres H, Marielle C, Monties B, Pollet B, Lapierre C. Lignin structure in Buxus sempervirens reaction wood. Phytochemistry 1997; 44(1): 35-39. http://dx.doi. org/10.1016/S0031-9422(96)00499-2

Bamber RK. A general theory for the origin of growth stresses in reaction wood: how trees stay upright. International Association of Wood Anatomists Journal 2001; 22(3): 205-212.

Bleile K. Vorkommen und Analyse von Zugholz bei Buche (Fagus sylvatica L.) als Ursache von Spannungen im Rundholz und Verwerfungen des Schnittholzes [thesis]. Freiburg: Universidade de Freiburg; 2006. 174 p.

Bowling AJ, Vaughn KC. Immunocytochemical characterization of tension wood: gelatinous fibers contain more than just cellulose. American Journal of Botany 2008; 95(6): 655-663. PMid:21632390. http:// dx.doi.org/10.3732/ajb.2007368

Clair B, Alméras T, Pilate G, Jullien D, Sugiyama J, Riekel C. Maturation Stress Generation in Poplar Tension Wood studied by Synchrotron Radiation Microdiffraction. Plant Physiology 2010; 152(3): 16501658. PMid:20071605 PMCid:2832242. http://dx.doi. org/10.1104/pp.109.149542

Clair B, Ruelle J, Beauchêne J, Prévost MF, Fournier M. Tension wood and opposite wood in 21 tropical rain forest species: occurrence and efficiency of the G-layer. International Association of Wood Anatomists Journal 2006; 27(3): 329-338.

Diaz-vaz JE, Ananías RA, Rodriguez S, Torres M, Fernández A, Poblete H. Compression wood in Pinus radiata II:density and chemical composition. Maderas, Ciencia y Tecnologia 2009; 11(2): 139-152.

Dunham RA, Cameron AD. Crow, Stem and Wood properties of Wind-damaged and undamaged Sitka spruce. Forest Ecology and Management 2000;
(135): $\quad 73-81 . \quad$ http://dx.doi.org/10.1016/S03781127(00)00299-1

Ferreira S, Lima JT, Trugilho PF, Monteiro TC. Excentricidade da medula em clones de Eucalyptus cultivados em diferentes topografias. Cerne 2008; 14(4): 335-340.

Grosser D. Defeitos de Madeira. Curitiba: Fupef; 1980. 62 p. (Série Técnica, n. 2).

Heinrich I, Gärtnert H. Variations in tension woodbroadleaved tree species in response to different mechanical treatments: implications for dendrochronology and mass movement studies. International Journal of Plant Sciences 2008; 169(7): 928-936. http://dx.doi. org/10.1086/589695

Jin $\mathrm{H}$, Kwon M. Mechanical bending induces tension wood formation with reduced lignin biosynthesis in Liriodendron tulipifera. Journal of Wood Science 2009; 55. (6): 401-408. http://dx.doi.org/10.1007/s10086-0091053-1

Klock U, Muñiz GIB, Hernandez JÁ, Andrade AS. Química da madeira. 3. ed. Curitiba: FUPEF; 2005. 85 p.

Kollman FFP, Côté WA. Principles of wood science and technology. New York: Springer-Verlag; 1968. v. 1, 592 p. http://dx.doi.org/10.1007/978-3-642-87928-9

Kübler H. Growth stresses in trees and related wood properties. Forestry-Abstracts 1987; 48(3): 131-189.

Kwon M. Lignan biosynthesis: implications for cell wall lignifications and secondary xylem formation in woody plants [thesis]. Washington State University; 2000. 246 p.

Latorraca JVF, Albuquerque CEC. Efeito do rápido crescimento sobre as propriedades da madeira. Floresta e Ambiente 2000; 7(1): 279-291.

Manso MM. Biomass estimation in the forest - a FONDEF project in Chile as a case of study [thesis]. Freiburg: Universidade de Freiburg; 2003.

Mattheck C, Kübler H. Wood - the internal optimization of trees. Berlin: Springer Verlag; 1998. v. 1, 129 p.

Monteiro TC, Silva RV, Lima JT, Baraúna EEP, Carvalho DM, Lima MT. Influência do lenho de tração nas propriedades físicas da madeira de Eucalyptus sp. Journal of Biotechnology and Biodiversity. Gurupi 2010; 1(1): 6-11.

MorohoshiN, Sakakibara A. Thechemical composition of reaction wood I. Mokuzai Gakkaishi 1971; 17: 393-399.

Oliveira JTS, Braz RL, Motta JP, Duarte APC, Rosado AM. Ações de ventos em povoamentos florestais. In: Chichorro JF, Garcia GO, Bauer MO, Caldeira MVW. Tópicos em Ciências Florestais. Alegre: Suprema, 2010. cap. 17 , p. 443-476.

Okuyama T, Yamamoto H, Yoshida M, Hattori Y, Archer RR. Growth stresses in tension wood - role 
of microfibrils and lignification. Annales des Science Forestière 1994; 51(3): 291-300. http://dx.doi. org/10.1051/forest:19940308

Pelz S. Eigenschaften und Verwendung des Holzes der Europäischen Lärche (Larix decidua Mill. ) unter besonderer Berücksichtigung des Reaktionsholzes [thesis]. Freiburg: Universidade de Freiburg; 2002. 279 p.

Panshin AJ, De Zeeuw C. Textbook of technology. 4th ed. New York: McGraw Hill; 1980. 705 p.

Peña SV, Peris FJJ. Tecnología de la Madera. Madrid: Ministerio de Agricultura Pesca y Alimentación - MAPA; 1996. 602 p.

Rodrigues J, Graça J, Pereira H. Influence of tree eccentric growth on syringyl/guaiacyl ratio in Eucalyptus globules wood lignin assessed by analytical pyrolisis. Jounal of Analytical and Applied Pyrolysis 2001; (58-59): 481-489.

Sousa LC. Caracterização da madeira de tração em Eucalyptusgrandis e sua influência na produção de polpa celulósica [dissertação]. Viçosa: Universidade Federal de Viçosa; 2004. 2004. 77 p.

Tarmian A, Remond R, Faezipour M, Karimi A, Perré P. Reaction wood drying kinetics: tension wood in Fagus sylvatica and compression wood in Picea abies. Wood Science and Technology 2008; 43(1-2): 113-130. http:// dx.doi.org/10.1007/s00226-008-0230-5

Timell TE. The chemical composition of tension wood. New York; 1969. p. 173-181.

Timell TE. Compression Wood in Gyminosperms. New York: Springer-Verlag; 1986. v. 1, 706 p.

Tisoumis GT. Wood as raw material: Source, Structure, Chemical Composition, Growth, Degradadion and Identification. New York: Pergamon Press; 1978. 276 p.
Tomazello Filho M. Análise da madeira de compressão em Pinus oocarpa: estrutura anatômica e quantificação. Boletim técnico IPEF 1987; (37): 61-68.

Tomazello Filho M, Silva DA. Formação e caracterização da madeira de compressão em Pinus caribaea var. hondurensis. Boletim técnico IPEF 1987; (37): 51-59.

Wang Y, Gril J, Clair B, Minato K, Sugiyama J. Wood properties and chemical composition of the eccentric growth branch of Viburnum odoratissimum var. awabuki. Trees 2010; 24(3): 541-549. http://dx.doi. org/10.1007/s00468-010-0425-X

Warensjö M. Compression wood in Scots pine and Norway spruce: distribution in relation to external geometry and the impact on the dimensional stability in sawn wood [thesis]. Swedish University of Agricultural Science; 2003.

Washusen R, Ades P, Evans R, Ilic J, Vinden P. Relationships between density, shrinkage, extractives content and microfibril angle in tension wood from three provenances of 10-year-old Eucalyptus globulusLabill. Holzforschung 2001; 55: 176-182. http:// dx.doi.org/10.1515/HF.2001.029

Washusen RK. The occurrence and characteristics of tension wood and associated wood properties in Eucalyptus globulus Labill [thesis]. Melbourne: University of Melbourne; 2000. 254 p.

Yasuda S, Sakakibara A. The chemical composition of lignin from compression wood. Mokuzai Gakkaishi 1975; 21: 363-369.

Zobel BJ, Van Buijtenen JP. Wood variation, its causes and control. New York: Springer-Verlarg; 1989. 337 p. http:// dx.doi.org/10.1007/978-3-642-74069-5 